Claremont Colleges

Scholarship@ Claremont

All HMC Faculty Publications and Research

HMC Faculty Scholarship

$1-1-2005$

\title{
A Theoretical Investigation of Charge Transfer in Several Substituted Acridinium Ions
}

Jason Lappe '00

Harvey Mudd College

Robert J. Cave

Harvey Mudd College

Marshall D. Newton

Brookhaven National Laboratory

I.V. Rostov

Brookhaven National Laboratory

\section{Recommended Citation}

Lappe, J, Cave, RJ, Newton, MD, Rostov, IV, “A Theoretical Investigation of Charge Transfer in Several Substituted Acridinium Ions," J. Phys. Chem. B. 2005, 109 (14): 6610. DOI: 10.1021/jp0456133

This Article is brought to you for free and open access by the HMC Faculty Scholarship at Scholarship @ Claremont. It has been accepted for inclusion in All HMC Faculty Publications and Research by an authorized administrator of Scholarship @ Claremont. For more information, please contact scholarship@cuc.claremont.edu. 


\title{
A Theoretical Investigation of Charge Transfer in Several Substituted Acridinium Ions ${ }^{\dagger}$
}

\author{
Jason Lappe and Robert J. Cave*,: \\ Department of Chemistry, Harvey Mudd College, Claremont California 91711 \\ Marshall D. Newton*, and I. V. Rostov" \\ Department of Chemistry, Brookhaven National Laboratory, Upton, New York 11973 \\ Received: September 27, 2004; In Final Form: November 23, 2004
}

\begin{abstract}
We present calculations for various properties of the ground and excited states of several arylamine-substituted acridinium ion systems that have been studied experimentally. Using ab initio and semiempirical quantum mechanical methods together with the generalized Mulliken-Hush $(\mathrm{GMH})$ model, we examine the excitation energies, dipole moment shifts, and electronic coupling elements for the vertical charge shift (CSh) processes in these systems. We also examine solvent effects on these properties using a dielectric continuum reaction field model. The results are in generally good agreement with available experimental results and indicate that there is strong electronic coupling in these systems over a wide range of torsional angles. Nevetheless, the initial and final cationic states remain reasonably well-localized over this range, and thus TICT state formation is unlikely in these systems. Finally, a version of the GMH model based on Koopmans' Theorem is developed and found to yield coupling elements generally within a factor of 2 of the many-electron GMH for a sample acridinium system, but with overestimated adiabatic and diabatic dipole moment differences.
\end{abstract}

\section{Introduction}

The understanding and control of photoinduced electron transfer (piet) continues to pose important challenges, driven especially by the need for fundamental mechanistic insight and the ultimate goal of improved solar energy conversion. ${ }^{1}$ Among different classes of piet, major effort has been focused on socalled charge separation (CS) processes of generic type

$$
\mathrm{D}^{*} \mathrm{~A} \rightarrow \mathrm{D}^{+} \mathrm{A}^{-}
$$

or

$$
\mathrm{DA}^{*} \rightarrow \mathrm{D}^{+} \mathrm{A}^{-}
$$

where the donor (D) and acceptor (A) moieties are chargeneutral, with one of them in a locally excited state generated by prior photoexcitation. ${ }^{2}$ Typically, the $\mathrm{D}$ and A sites may be separated by a molecular spacer or bridge (DBA), but for compactness our notation will suppress the B site. Processes $1 \mathrm{a}$ and $1 \mathrm{~b}$ may be either intermolecular or intramolecular, with the latter case offering the advantage of good control of D/A interaction geometry (both separation distance and relative orientation).

While piet (eq 1) may be thermal (if the photoexcited species (D* or A*) are thermally equilibrated), the same final CS state may often be obtained directly from the ground state by the optical process: ${ }^{3}$

$$
\mathrm{DA} \underset{h v}{\longrightarrow} \mathrm{D}^{+} \mathrm{A}^{-}
$$

\footnotetext{
† Part of the special issue "David Chandler Festschrift".

* Corresponding authors.

E-mail: Robert_Cave@hmc.edu.

\&-mail: Newton@bnl.gov.

" Present address: Supercomputing Facility, Australian National University, Canberra, ACT 0200, Australia.
}

Another important class of piet processes is that of the charge shift (CSh) type, ${ }^{4,5}$ which, analogous to eqs 1 and 2, may be thermal, after photoexcitation:

$$
\begin{aligned}
& \mathrm{D}\left(\mathrm{A}^{+}\right)^{*} \rightarrow \mathrm{D}^{+} \mathrm{A} \\
& \mathrm{D}^{*} \mathrm{~A}^{+} \rightarrow \mathrm{D}^{+} \mathrm{A}
\end{aligned}
$$

or optical:

$$
\mathrm{DA}^{+} \underset{h v}{\longrightarrow} \mathrm{D}^{+} \mathrm{A}
$$

where a charge-neutral electron donor interacts with a cationic acceptor (alternatively, one could consider CSh involving an anionic donor and a charge-neutral acceptor). The examples of CSh considered below are of the optical intramolecular type (eq 4): $\mathrm{D}_{\text {and }} \mathrm{A}^{+}$are closed-shell moieties and $\mathrm{D}^{+}$and $\mathrm{A}$ are, respectively, local charged and neutral radical sites, with overall singlet spin coupling.

In modeling CS behavior, including solvatochromic trends in media of variable polarity, the Onsager model based on a point dipole of variable magnitude and direction in a spherical cavity has been found quite useful. ${ }^{6,7}$ Furthermore, the degree of charge localization of the D and A sites in the CS state (and hence the magnitude of the CS dipole moment) may depend on torsional relaxation with respect to single bonds in the DBA system. ${ }^{8}$ In the extensively studied "twisted intramolecular charge transfer" (TICT) process, polar solvation promotes the twisting of $\pi$ type D and A groups toward an orthogonal conformation, thereby minimizing D/A overlap and maximizing charge separation.

In the simple limiting case of CS, the initial state (DA) dipole moment is zero or much smaller than that for the final state $\left(\mathrm{D}^{+} \mathrm{A}^{-}\right)$dipole. In practice, less clear-cut situations occur (e.g., as in the case of optical CS in coumarin dyes $\left.{ }^{9}\right)$. Of course, the 
reverse type of charge transfer (CT) may occur (charge recombination $(\mathrm{CR})^{2,10}$ ), in which the initial state has the higher degree of charge separation (e.g., as for absorption in betaine dyes $^{6,7}$ ).

In the case of CSh processes (eqs 3 and 4), analogous questions arise concerning charge distributions and the potential role of torsions in controlling them. Nevertheless, the situation is quite distinct, and a simple point dipole Osager model is not directly relevant for modeling solvent effects. While the change in dipole moment is still very significant, the individual state dipoles $\left(\mathrm{D}^{+} \mathrm{A}\right.$ and $\left.\mathrm{DA}^{+}\right)$, while well-defined, are now dependent on coordinate origin because of the net molecular charge. As a result, a two-sphere Born-type model (based on point monopoles, i.e., charges, instead of dipoles $)^{11}$ may be more useful, with the effective radii of the $\mathrm{D}$ and $\mathrm{A}$ sites at least qualitatively indicating the relative solvation stabilization (i.e., solvent contribution to the net free energy difference, $\Delta G^{\circ}$ ) of the $\mathrm{DA}^{+}$ and $\mathrm{D}^{+} \mathrm{A}$ states.

In optical charge transfer (both CS and CSh type), which is a Franck-Condon process generally occurring at the equilibrium configuration of the initial state, the excitation energetics involve not only $\Delta G^{\circ}$ but also the reorganization energy $\lambda$ (one-half the Stokes shift separating absorption and emission peaks),

$$
h v_{\max }=\Delta G^{\circ} \pm \lambda
$$

where \pm corresponds, respectively, to absorption and emission. ${ }^{12}$ Equation 5 pertains to an electronic system linearly coupled to a harmonic "bath" (intramolecular and/or medium modes). For et in polar media, eq 5 corresponds to standard Marcus theory, in which $\lambda$ contains additive intramolecular and medium polarization contributions. ${ }^{13,14}$ Several comparative studies of $\mathrm{CS}, \mathrm{CR}$, and CSh processes have been reported in the literature. ${ }^{15-18}$

In the present study, we examine optical intramolecular CSh in a set of $\mathrm{DA}^{+}$systems composed of a 10-methyl acridinium acceptor (A) and several amino-phenyl (or biphenyl) donors (D) attached at the acridinium $\mathrm{C}_{9}$ position (Figure 1). These systems were selected in view of prior experimental investigations of amino-aryl (and amido-aryl) acridinium ions by Verhoeven et al. ${ }^{19}$ and Jones and Wasieleski et al., ${ }^{5,20}$ in which the nature of the CSh state was probed by steady-state absorption and emission and transient absorption in different solvents. ${ }^{21}$ These studies supported a model based on the three states involved in eqs $3 \mathrm{a}$ and 4 , in which electron-donating substituents on the aryl donor group (and also an unsubstituted naphthyl donor group) place the $\mathrm{D}^{+} \mathrm{A}$ CSh state below the locally exited $\mathrm{D}\left(\mathrm{A}^{+}\right)^{*}$ state and above the ground $\mathrm{DA}^{+}$state.

Most of the present report deals with the reference vertical absorption behavior of the gas-phase species displayed in Figure 1 , including dependence on the torsional angles (denoted $\varphi, \gamma$, and $\omega$ ). Single-excitation configuration interaction (CI) calculations (CIS) using ab initio or semiempirical Hamiltonians were employed, based on ab initio or density functional theory (DFT) ground-state geometries. Some results for vertical absorption in solution, based on a reaction field (RF) semiempirical CIS treatment, are also presented and discussed in terms of calculated trends with solvation. The transition from ground $\left(\mathrm{DA}^{+}\right)$to $\mathrm{CSh}$ $\left(\mathrm{D}^{+} / \mathrm{A}\right)$ state is characterized in terms of excitation energy $\left(\Delta E_{12}\right.$, corresponding to $h v_{\max }$ in eq 5), dipole moment shift $\left(\Delta \mu_{12}\right)$, and transition moment $\left(\mu_{12}\right)$, where labels 1 and 2 denote the adiabatic states defining the two-state model employed in the present work (in standard spectroscopic notation these are the $\mathrm{S}_{0}$ and $\mathrm{S}_{1}$ states, the two lowest lying of the set of three states

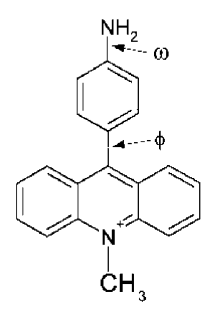

ap-ac

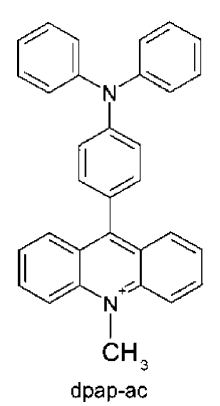

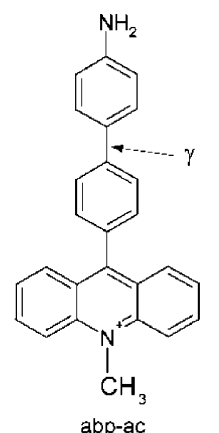

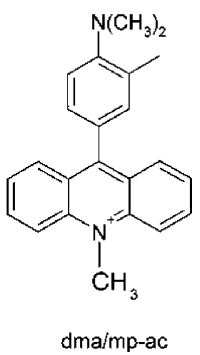

Figure 1. The four structures are 9-aryl,10-methyl derivatives of the acrdinium cation, where the labels ap, dpap, dma/mp, and abp denote, respectively, 4-aminophenyl, 4- $N, N$-diphenylaminophenyl, 4- $N, N$-dimethyl,3-methylaminophenyl, and 4'-amino-4-biphenylyl. The dihedral angles associated with the single bonds linking the phenyl groups are labeled $\varphi$ (phenyl/acridinium) and $\gamma$ (the biphenylyl moiety), with zero defined by coplanarity (as drawn). For the aminophenyl linkage (NC), where $\mathrm{X}_{2} \mathrm{~N}$ is the amino group, $\omega$ refers to the dihedral angle between the $\mathrm{XNC}$ and $\mathrm{NCC}^{\prime}$ planes, associated with the $\mathrm{XNCC}^{\prime}$ bonded sequence $\left(\mathrm{X}=\mathrm{H}\right.$ (ap-ac and abp-ac) or $\mathrm{C}\left(\mathrm{dpap}-\mathrm{ac}\right.$ and dma/mp-ac)). The $\mathrm{X}_{2} \mathrm{~N}$ moiety is not in general planar, nor is it symmetrically disposed with respect to the mean plane of the attached phenyl group. Thus, two distinct $\omega$ values are presented in Table 1 (based on the two respective $\mathrm{XNC}$ planes). Distinctions due to slight nonplanarity of the phenyl-N moiety are neglected by averaging to yield a mean plane. With this averaging, either of the two possible choices for $\mathrm{C}^{\prime}$ yield the same results.

discussed above). Using the generalized Mulliken-Hush model (GMH) ${ }^{22-25}$ we obtained the corresponding diabatic state quantities, including the effective D/A Hamiltonian coupling element, $H_{\mathrm{DA}}$, and the dipole moment shift, $\Delta \mu_{\mathrm{DA}}$, from the calculated adiabatic (spectroscopic) state results, as discussed in the next section. A point of particular interest is the variation of $H_{\mathrm{DA}}$ (non-Condon behavior) ${ }^{26,27}$ with respect to torsion angles and degree of solvation. Here the site labels D and A are used to denote the diabatic states: that is, the initial and final diabatic states are identified by the sites on which the cationic charge is primarily localized (respectively, A and D). The picture in terms of many-electron D and A states may be replaced (to good approximation) by a one-electron orbital counterpart (i.e., effective D and A orbitals), if the manifold of nontransferring electrons is represented by an invariant effective core. ${ }^{28}$ Examples of one-electron models are considered below.

Strictly speaking, eq 5, based on linear coupling of solute and solvent, generally refers to charge-localized diabatic states, assumed not to vary with solvent reaction coordinate (such an effect may arise because of solute polarizability, as discussed in Section 4). Since the adiabatic states obtained in the calculations reported here remain fairly localized, eq 5 provides a useful reference for assessing trends in adiabatic transitions. 


\section{Theoretical Methods}

In all cases the equilibrium geometries were obtained from geometry optimizations using DFT with the B3LYP exchange correlation functional and the $6-31 \mathrm{G}^{*}$ basis set using Gaussian $98 .^{29}$

Excitation energies from the SCF ground state to the two lowest singlet excited states were obtained using CI at the CIS level. For the ab initio calculations, excitations were allowed from all noncore orbitals into all virtual orbitals. In the CIS calculations based on the semiempirical INDO/s Hamiltonian, ${ }^{30}$ we allowed excitations from the highest 11 occupied orbitals into the lowest 11 virtual orbitals. In a test calculation, only small changes in the INDO/s CIS excitation energies (less than a few percent) were obtained when the number of orbitals was increased. The INDO/s CIS calculations were performed using the Zerner code, ${ }^{31}$ and ab initio CIS calculations were performed using Gaussian 98. ${ }^{29}$ Results for RF calculations are described in Section 4.

To examine the sensitivity of our results to the choice of ground-state geometry, as well as to investigate the effects of thermal averaging, we performed calculations at several geometries based on rotations about one or more bonds, relative to the optimized ground-state geometry. Beginning at the groundstate optimized geometry, we rotated the two ends of the molecule about the angle(s) indicated in Figure 1. For the $\varphi$ coordinate, we chose values of $10,30,70$, and $90^{\circ}\left(90^{\circ}\right.$ corresponds to a perpendicular orientation of the ring planes) in addition to the equilibrium geometry, where $\varphi$ is about $50^{\circ}$. In each case all bond lengths and all other angles were maintained at their values for the equilibrium geometry. At each value of $\varphi$, we evaluated the total energy using DFT (B3LYP/ 6-31G*) and ab initio RHF theory (6-31G*). In the case of abpac we also investigated the effects of rotation with respect to $\gamma$. In this case, we chose values of $10,50,70$, and $90^{\circ}$ in addition to the equilibrium value. Once again, all bond lengths and all other angles were constrained to their values for the equilibrium structure. At each of these geometries excited-state calculations were also performed, as described above.

In addition to excitation energies $\left(\Delta E_{12}\right)$, we report adiabatic dipole moment differences $\left(\Delta \mu_{12}\right)$ and transition dipole moments $\left(\mu_{12}\right)$ between the ground and CSh states, and the electronic coupling element $\left(H_{\mathrm{DA}}\right)$ connecting the diabatic ground and CSh states and the corresponding diabatic dipole moment shift $\left(\Delta \mu_{\mathrm{DA}}\right)$. On the basis of use of the two-state GMH approach, ${ }^{22-25}$ these latter diabatic quantities may be expressed in terms of adiabatic quantities as follows:

$$
\begin{gathered}
H_{\mathrm{DA}}=\mu_{12} \Delta E_{12} /\left[\left(\Delta \mu_{12}\right)^{2}+4\left(\mu_{12}\right)^{2}\right]^{1 / 2} \\
\Delta \mu_{\mathrm{DA}}=\left[\left(\Delta \mu_{12}\right)^{2}+4\left(\mu_{12}\right)^{2}\right]^{1 / 2}
\end{gathered}
$$

The quantity $\Delta \mu_{\mathrm{DA}}$ provides an objective definition of the mean D/A separation, $r_{\mathrm{DA}} \equiv \Delta \mu_{\mathrm{DA}} /$ e (i.e., the separation of the centroids of the effective D and A orbitals), where e is the magnitude of the electronic charge. Note that the scalar quantity $\mu_{12}$ in this work is the projection of the transition dipole vector in the direction defined by the $\Delta \mu_{12}$ vector. ${ }^{22}$ In all cases examined, the two vectors were found to be closely aligned, nearly coinciding with the dominant molecular axis (see Figure 1). In the GMH approach, the diabatic states are defined by maximizing $\Delta \mu_{\mathrm{DA}}$, leading to $\mu_{\mathrm{DA}}=0 .{ }^{22-25}$

In the current instance, we assume that the diabatic states of interest are adequately accounted for within the space defined by the pair of lowest adiabatic states, although the GMH
TABLE 1: Optimized Dihedral Angles ${ }^{a}$

\begin{tabular}{lccl}
\hline system & $\varphi$ & $\gamma$ & \multicolumn{1}{c}{$\omega^{b}$} \\
\hline ap-ac & 54 & & $10 / 172$ \\
abp-ac & 56 & 29.5 & $18 / 164$ \\
dma/mp-ac & 56 & & $20 /-124$ \\
dpap-ac & 52 & & $19 /-161$
\end{tabular}

${ }^{a}$ Based on DFT calculations and averaged over the pair of choices (arising from the slight nonplanarity of the phenyl rings) for the given dihedral angle definition (see Figure 1 caption). The individual choices differed by at most $1^{\circ} .{ }^{b}$ The distinct $\omega$ values are defined in the Figure 1 caption. Angle pairs of the same (opposite) sign involve $X$ groups on the same (opposite) side of the mean plane of the attached phenylnitrogen moiety.

approach is not inherently limited to the two-state limit. ${ }^{22-25}$ Test calculations in vacuo indicate that multistate effects are modest in these systems, ${ }^{24}$ and this situation is also observed when solvent is included, as reported in Section 4.

We also present results (in Section 5) testing a simplified one-electron version of GMH, which we designate as the Koopmans' Theorem ${ }^{32}$ GMH model (KT-GMH). Relative to an $n$-electron closed-shell SCF ground-state reference system, one can use the method to treat optical (or reverse) electron transfer in the same $n$-electron system (where the transfer involves an occupied and a virtual orbital), or alternatively, hole transfer in the $(n-1)$-electron system (using pairs of occupied orbitals) or electron transfer in the $(n+1)$-electron system (using pairs of virtual orbitals). Voityuk and Rösch compared the KTGMH method with their fragment charge difference method for calculating the electronic coupling for hole transfer. ${ }^{33}$ Here we compare KT-GMH results with GMH results based on CI wave functions for the ground and excited states.

Given the low energy associated with torsional motion in these systems, we have chosen to present approximate thermally averaged excitation energies, dipole and transition dipole moments, and coupling elements, using the expression:

$$
\begin{array}{r}
X_{\mathrm{av}}=P\left\{2 \sum X\left(\theta_{i}\right) \exp \left[-E\left(\theta_{i}\right) / k T\right]+\right. \\
X(90) \exp [-E(90) / k T]\}
\end{array}
$$

where $X$ is the quantity of interest, $P$ is the constant needed to normalize the Boltzmann weighting factors, and $\theta$ stands for the torsion angle of interest $(\varphi$ or $\gamma$ ). We assume that $E(\theta)$ is symmetric about $90^{\circ}$ in view of the near-planar structures of the individual subunits, and $\theta_{i}$ samples $\theta$ within the range $0^{\circ}<$ $\theta<90^{\circ}$, as described above.

Finally, to more completely define the character of the CSh states obtained for the acridinium systems described above, we also examined a derivative of ap-ac where the $\mathrm{N}^{+}$on the acridinium was replaced by the isoelectronic $\mathrm{C}$ atom. The geometry for this system was obtained via a DFT (B3LYP/6$\left.31 \mathrm{G}^{*}\right)$ geometry optimization, and excitation energies at selected geometries were obtained using INDO/s CIS.

\section{Gas-Phase Results}

3.1. Ground-State Geometry. In Table 1, we present selected information regarding the equilibrium geometries for the species ap-ac, abp-ac, dpap-ac, and dma/mp-ac (see Figure 1). Complete geometry information is provided in the Supporting Information, but here we focus on the dihedral angles between the various subunits of each compound. The angle $\varphi$ is the dihedral angle connecting the acridinium and the phenyl ring. DFT predicts that $\varphi$ is within $2^{\circ}$ of $54^{\circ}$ for all four compounds. These values may be compared with the range of values $\left(70 \pm 10^{\circ}\right)$ observed for several aryl-acridinium crystal structures. ${ }^{34}$ The compound 
TABLE 2: Ap-ac: Calculated CSh Results and $\varphi$-Dependence ${ }^{a}$

\begin{tabular}{|c|c|c|c|c|}
\hline geometry $^{b}$ & $H_{\mathrm{DA}}\left(10^{3} \mathrm{~cm}^{-1}\right)$ & $\Delta \mu_{12}(\mathrm{D})$ & $\mu_{12}(\mathrm{D})$ & $\Delta E_{12}(\mathrm{eV})$ \\
\hline \multicolumn{5}{|c|}{ ab initio } \\
\hline opt & 12.4 & 9.03 & 6.38 & 3.75 \\
\hline$\varphi=90^{\circ}$ & 0.80 & 16.84 & 0.48 & 3.68 \\
\hline ave (DFT) & 12.2 & 9.24 & 6.40 & 3.75 \\
\hline ave (RHF) & 11.2 & 10.41 & 5.89 & 3.74 \\
\hline \multicolumn{5}{|c|}{ INDO/s } \\
\hline opt & 7.5 & 13.29 & 6.64 & 2.62 \\
\hline$\varphi=90^{\circ}$ & 0.39 & 18.43 & 0.39 & 2.36 \\
\hline ave (DFT) & 7.3 & 13.47 & 6.53 & 2.61 \\
\hline ave (RHF) & 6.6 & 14.46 & 5.92 & 2.57 \\
\hline experiment $^{c}$ & 4.4 & 17.3 & 4.45 & 2.38 \\
\hline
\end{tabular}

${ }^{a}$ Calculated CSh results for vertical CSh (ground state $\left(\mathrm{S}_{0}\right)$ to $\mathrm{CSh}$ state $\left(\mathrm{S}_{1}\right)$ ), based on single excitation configuration interaction (CIS) using ground-state SCF (RHF) orbitals at the ab initio $\left(6-31 \mathrm{G}^{*}\right.$ basis) or semiempirical (INDO/s) level. ${ }^{b}$ The calculations employed the ground-state optimized (opt) geometry, obtained at the DFT (B3LYP) level of theory. For the torsion angle $\varphi$ (Figure 1), a Boltzmann average (ave) was carried out, based on DFT and ab initio (RHF) results, and the limiting case of $\varphi=90^{\circ}$ was also considered (keeping all other coordinates fixed at ground-state equilibrium values). The minimum energy with respect to $\varphi$ in the $\mathrm{S}_{1}$ state occurred at $70^{\circ}$ on the grid used here. ${ }^{c}$ Experimental spectral data (measured in methanol solution and 2-methyl tetrahydrofuran/butyronitrile glasses) are from Vance, Johnson, and Hupp. ${ }^{37}$ Uncertainties in $\Delta \mu_{12}$ values are estimated to be $\sim 10 \%$, based on results obtained for abp-ac (see Tables 3 and 4).

abp-ac has another angular degree of freedom (angle $\gamma$, between the rings in the biphenyl group). It was found to be approximately $30^{\circ}$ here, which is $10-15^{\circ}$ smaller than that found in biphenyl itself. ${ }^{35}$ To examine whether this was an artifact of the method used, we performed a geometry optimization for biphenyl at the same DFT level (B3LYP exchange correlation functional and the 6-31G* basis) and found a dihedral angle of $40^{\circ}$, suggesting that the method is reasonably accurate in describing this feature and that the smaller angle found in the acridinium compound may be a reliable result.

The dihedral angles involving the terminal amines reflect a significant degree of pyramidalization of the amine nitrogen (except for dpac-ac) and also appreciable twisting of the amine group (when dimethyl or diphenyl substituted) with respect to the mean plane of the attached phenyl ring.

3.2. CSh Results for ap-ac. In Table 2, we offer detailed results for vertical CSh in ap-ac. In particular, we present results for the electronic coupling element $\left(H_{\mathrm{DA}}\right)$, the magnitudes of the adiabatic dipole moment difference, the projected transition dipole moment, and the electronic excitation energy. For each quantity, we present results based on four different geometries (equilibrium value for $\varphi$, designated "vertical"; $\varphi=90^{\circ}$ such that the two ring planes are perpendicular; and two different thermal averages, one based on ground-state DFT (B3LYP) energies, the other on ground-state ab initio (RHF) energies). In addition, we present results based on two distinct CIS excitedstate methods, ab initio and semiempirical (INDO/s).

Considering the ab initio CIS results first, it is seen that the coupling element and transition dipole moment are quite large at the equilibrium geometry, and they are both quite sensitive to changes in the angle $\varphi$. At $\varphi=90^{\circ}$, the coupling element and the transition dipole moment drop considerably. One might expect that the coupling would be exactly zero at this geometry, but small departures from planarity of the rings mean that this angle will yield a near zero, but not exactly zero value. In exploratory calculations (not shown), we have found that the coupling element rises quite steeply from its value at $\varphi=90^{\circ}$, such that even at $70^{\circ}$ the coupling is $70 \%$ of that at the equilibrium value, and that further decreases in $\varphi$ do not lead to substantial increases in the coupling. This large value of the coupling element is what gives rise to the considerable sensitivity of the adiabatic dipole moment difference to geometry as well. At $\varphi=90^{\circ}$, the orbitals involved in the transition from the ground state to the CSh state are strongly localized on different portions of the compound, one on the acridinium, the other on the arylamine group. Since the coupling is relatively small, the adiabatic and diabatic states are rather similar, and the adiabatic dipole moment difference is comparable to the diabatic difference. While the diabatic states do not change significantly as the angle $\varphi$ decreases (and the coupling increases), the adiabatic states (mixed via the large coupling) do change, because of substantial mixing of the diabatic states. The limit of such mixing would be an adiabatic dipole moment difference of zero, but this limit is not approached here because of the difference in energy between the diabatic states. Nevertheless, the mixing is significant enough to lead to a decrease in the adiabatic dipole moment difference of more than $7 \mathrm{D}$ in proceeding to the equilibrium geometry from the $\varphi=$ $90^{\circ}$ structure. Note that the thermally averaged vertical CSh properties of ap-ac (and of the other systems dealt with below) do depart significantly from their values based on the groundstate equilibrium geometry.

It should be emphasized that the separation of centroids associated with the cationic charge in the initial and final states of the CSh process (whether estimated in terms of $\Delta \mu_{12}$ or $\left.\Delta \mu_{\mathrm{DA}}\right)$ is considerably smaller than the overall length of the DA molecule (typically $\sim 50 \%$ of the distance separating the $\mathrm{N}$ atoms in ap-ac and the other acridinium systems considered below).

The INDO/s CIS results for ap-ac are qualitatively quite similar to the ab initio CIS results, with some quantitative differences. The INDO/s transition dipole moments at a given geometry are quite similar to the ab initio values, as is the difference between $\Delta \mu_{12}$ at the equilibrium geometry and $\varphi=$ $90^{\circ}$ geometry. However, the INDO/s results predict significantly lower excitation energies, somewhat larger $\Delta \mu_{12}$ values, and $H_{\mathrm{DA}}$ values that are smaller than those from the ab initio CIS calculations by nearly a factor of 2 . Trends similar to those obtained for the ab initio CIS results in the comparison of fixed geometry and thermally averaged values are observed for INDO/ s. Clearly either theoretical method predicts quite large coupling elements connecting the initial and final diabatic states for this system. Such coupling would be expected to promote facile CR $\left(\mathrm{D}^{+} \mathrm{A} \rightarrow \mathrm{DA}^{+}\right),{ }^{36}$ although this reverse $\mathrm{CSh}$ process would most likely involve the relaxed CSh molecular structure. Thus, refined estimates of $H_{\mathrm{DA}}$ for $\mathrm{CR}$ would require full optimization of the $\mathrm{D}^{+} \mathrm{A}$ geometry.

3.2.1. Comparison with Experiment. The experimental values presented in Table 2 come from work by Hupp et al. ${ }^{37}$ The experimental coupling element is in better agreement with the INDO/s results but is still about $3000 \mathrm{~cm}^{-1}$ smaller than the theoretical value. In addition, the experimental adiabatic dipole moment is about 4D larger than the INDO/s value, the transition dipole moment is about 2D smaller than either theoretical value, and the experimental excitation energy, while in better agreement with INDO/s than with the ab initio values, is lower than either. Inspection of the INDO/s results from the equilibrium geometry and $\varphi=90^{\circ}$ geometry might lead one to believe that better overall agreement would be obtained were larger contributions from angles nearer $\varphi=90^{\circ}$ used (say $80^{\circ}$ to $90^{\circ}$ ), but results presented below indicate that there is no a priori reason to doubt the DFT torsional potential at this point. 
TABLE 3: Abp-ac: Calculated Results and $\varphi$-Dependence ${ }^{a}$

\begin{tabular}{lcccc}
\hline geometry $^{b}$ & $H_{\text {DA }}\left(10^{3} \mathrm{~cm}^{-1}\right)$ & $\Delta \mu_{12}(\mathrm{D})$ & $\mu_{12}(\mathrm{D})$ & $\Delta E_{12}(\mathrm{eV})$ \\
\hline \multirow{5}{*}{ opt } & 9.8 & 17.1 & 7.75 & 3.60 \\
$\varphi=90^{\circ}$ & 0.79 & 25.3 & 0.68 & 3.69 \\
ave (DFT) & 9.4 & 17.6 & 7.48 & 3.61 \\
ave (RHF) & 8.3 & 19.2 & 6.59 & 3.63 \\
\multicolumn{5}{c}{ INDO/s } \\
opt & 5.1 & 23.5 & 7.05 & 2.46 \\
$\varphi=90^{\circ}$ & 0.43 & 27.1 & 0.62 & 2.35 \\
ave (DFT) & 4.9 & 23.9 & 6.76 & 2.46 \\
ave (RHF) & 4.1 & 25.0 & 5.79 & 2.43 \\
experimental & 4.0 & $23.5 \pm 2.3$ & 4.61 & 2.73
\end{tabular}

${ }^{a}$ Quantities as defined in Table 2. ${ }^{b}$ The minimum energy value of $\varphi$ for the $\mathrm{S}_{1}$ state occurred at the ground state $\left(\mathrm{S}_{0}\right)$ value (Table 1$)$ on the grid used here.

Furthermore, the effects of solvent and the difficulty of unambiguously extracting the various input quantities to the GMH based on experimental data suggest that it would be premature to assume these experimental data represent the final word on these quantities. Rather, we believe the comparison indicates that reasonable agreement is obtained at this stage and that both theory and experiment lend strong support to the idea that large coupling obtains in these systems at thermally accessible torsion angles.

3.2.2. TICT. Systems of the present type (arylamines) have been frequently proposed as candidates for TICT state formation, based on twisting around the terminal amine torsional angles. ${ }^{8}$ We examined this possibility for ap-ac by rotating about the terminial amine, using RHF/CIS energies. Twisting by $40^{\circ}$ (relative to the symmetrical equilibrium local geometry at the amine) causes the $S_{1}$ state to rise $8.6 \mathrm{kcal} / \mathrm{mol}$ relative to the essentially coplanar minimum. Over the same angle variation the ground state rose $6.0 \mathrm{kcal} / \mathrm{mol}$, suggesting that the $S_{1}$ state (i.e., the $\mathrm{CSh}^{+} \mathrm{A}$ state) is even less likely than the ground state to twist about this coordinate. Furthermore, $\Delta \mu_{12}$ decreases as the terminal amine is rotated. Thus, on the basis of these in vacuuo results we expect that TICT state formation in polar media is unlikely for ap-ac. Exploratory calculations (not shown) suggest this is also the case for the other systems studied here.

3.2.3. TDDFT. For ap-ac, we also briefly examined the use of TDDFT based on the B3LYP functional and the adiabatic approximation. ${ }^{38}$ Other groups have used TDDFT to treat CT states and found quite low estimates for CT excitation energies. ${ }^{39-41}$ In the case of ap-ac, we found that TDDFT/ B3LYP gave excellent results for the CSh excitation energy at the equilibrium geometry, but at the $\varphi=90^{\circ}$ geometry it yields an excitation energy of about $1.3 \mathrm{eV}$, lower than experiment or INDO/s by about $1 \mathrm{eV}$. Of course, in these systems for $\varphi$ near its ground-state equilibrium value there is considerable overlap between the initial and final states (as attested to by the large coupling elements obtained above), but at the $\varphi=90^{\circ}$ geometry the overlap becomes quite small, and TDDFT fails, as one expects. As a result, we have not pursued its use for the other systems studied here, and we note that any applications of the method to weak-overlap CT interactions should be viewed with healthy skepticism.

3.3. CSh Results for Abp-ac. Similar results to those obtained for ap-ac are found for abp-ac, as shown in Table 3. As was true for ap-ac, the ab initio CIS electronic coupling element is larger than the INDO/s value, the transition dipole moments are similar for the two methods, and the ab initio excitation energies are considerably higher than those for INDO/ s. We note modest differences in comparison with ap-ac: the
TABLE 4: Abp-ac: Calculated CSh Results and $\gamma$-Dependence ${ }^{a}$

\begin{tabular}{lcccc}
\hline geometry $^{b}$ & $H_{\text {DA }}\left(10^{3} \mathrm{~cm}^{-1}\right)$ & $\Delta \mu_{12}(\mathrm{D})$ & $\mu_{12}(\mathrm{D})$ & $\Delta E_{12}(\mathrm{eV})$ \\
\hline \multirow{5}{*}{ opt } & 9.8 & 17.1 & 7.75 & 3.60 \\
ave (DFT) & 9.8 & 17.0 & 8.06 & 3.57 \\
ave (RHF) & 10.0 & 16.2 & 7.55 & 3.67 \\
& \multicolumn{5}{c}{ INDO/s } \\
opt & 5.1 & 23.5 & 7.05 & 2.46 \\
$\gamma=90^{\circ}$ & 0.16 & 38.6 & 0.26 & 2.99 \\
ave (DFT) & 5.1 & 23.5 & 7.04 & 2.46 \\
ave (RHF) & 5.1 & 23.8 & 6.86 & 2.52 \\
experimental & 4.0 & $23.5 \pm 2.3$ & 4.61 & 2.73
\end{tabular}

${ }^{a}$ Quantities as defined in Table 2, except that variations were carried out for torsion angle $\gamma$ (instead of $\varphi$, as in Table 3). The upper limit for $\gamma$ used in the averaging was $70^{\circ}$, since $90^{\circ}$ does not yield a CSh state within the first two excited states. ${ }^{b}$ The minimum energy value of $\gamma$ for the $\mathrm{S}_{1}$ state occurred at $10^{\circ}$ on the grid used here.

TABLE 5: Dma/mp-ac: Calculated CSh Results and $\varphi$-Dependence ${ }^{a}$

\begin{tabular}{lcccc}
\hline \multicolumn{1}{c}{ geometry } & $H_{\mathrm{DA}}\left(10^{3} \mathrm{~cm}^{-1}\right)$ & $\Delta \mu_{12}(\mathrm{D})$ & $\mu_{12}(\mathrm{D})$ & $\Delta E_{12}(\mathrm{eV})$ \\
\hline opt & 6.4 & 15.7 & 6.07 & 2.58 \\
$\varphi=90^{\circ}$ & 0.24 & 19.6 & 0.26 & 2.38 \\
ave $(\mathrm{DFT})$ & 6.1 & 16.0 & 5.85 & 2.57 \\
ave (RHF) & 5.2 & 17.1 & 4.99 & 2.52 \\
experimental & 3.0 & 20.2 & 2.89 & 2.66
\end{tabular}

${ }^{a}$ Quantities as defined in Table 2. Only INDO/s results are included.

INDO/s coupling element and adiabatic dipole moment shift are in excellent agreement with the experimental values ${ }^{37}$ but the experimental excitation energy is now above the INDO/s value.

3.3.1. $\gamma$-Dependence. Abp-ac represents a system with an extra degree of torsional freedom compared to the other systems studied here, that being the biphenyl torsional angle. Results that treat the effects of variation of this angle are presented in Table 4. The ground-state thermal averaging leads to values of the various quantities that are very similar to those for the equilibrium geometry. However, the INDO/s results for $\gamma=$ $90^{\circ}$ (biphenyl ring planes perpendicular) show that if the biphenyl rings were to become perpendicular the charge separation distance would increase considerably. In such a case the final hole state would be localized on the terminal phenylamine (in contrast to the situation for smaller angles, where the hole is delocalized over the two phenyl rings, leading to a shorter CT distance). In fact, however, optimization of $\gamma$ for the CSh state $\left(\mathrm{D}^{+} \mathrm{A}\right)$ leads to a structure closer to coplanarity than for the ground state. Thus, to the extent that geometrical relaxation is important, it will lead to somewhat smaller values of the adiabatic dipole moment shift than obtained at the ground-state equilibrium geometry.

3.4. CSh Results for Dma/mp-ac and dp-ac. Tables 5 and 6 present INDO/s results for dma/mp-ac and dpap-ac and comparison with experiment. Trends similar to those observed for the INDO/s results for ap-ac are obtained here. Once again, the calculated compling elements are somewhat larger than those obtained from experiment.

3.5. Ap-ac vs a Neutral Isoelectronic Variant. To further probe the energetics of these CSh systems, we created a system analogous to ap-ac, except for substitution of a $\mathrm{C}$ for the $\mathrm{N}^{+}$of the acridinium ring (yielding a substituted anthracene). One might expect a considerably raised CSh-like state (actually, the state is now a CS state, since the substituted anthracene is not 
TABLE 6: Dpap-ac: Calculated CSh Results and $\varphi$-Dependence ${ }^{a}$

\begin{tabular}{lcccc}
\hline \multicolumn{1}{c}{ geometry } & $H_{\mathrm{DA}}\left(10^{3} \mathrm{~cm}^{-1}\right)$ & $\Delta \mu_{12}(\mathrm{D})$ & $\mu_{12}(\mathrm{D})$ & $\Delta E_{12}(\mathrm{eV})$ \\
\hline opt & 5.6 & 19.4 & 7.48 & 2.28 \\
$\varphi=90^{\circ}$ & 0.01 & 23.2 & 0.09 & 2.02 \\
ave (DFT) & 5.6 & 19.4 & 7.44 & 2.27 \\
ave (RHF) & 5.1 & 20.2 & 6.79 & 2.23 \\
experimental & 3.3 & 22.1 & 4.27 & 2.28
\end{tabular}

${ }^{a}$ Quantities as defined in Table 2. Only INDO/s results are included.

TABLE 7: Calculated Solvatochromic Effects for Vertical CSh Absorption ${ }^{a}$

\begin{tabular}{lccccc}
\hline$\epsilon_{0} / \epsilon_{\infty}$ & $H_{\mathrm{DA}}\left(10^{3} \mathrm{~cm}^{-1}\right)$ & $\Delta \mu_{12}(\mathrm{D})$ & $\Delta \mu_{\mathrm{DA}}(\mathrm{D})$ & $\mu_{12}(\mathrm{D})$ & $\Delta E_{12}(\mathrm{eV})^{b}$ \\
\hline \multicolumn{5}{c}{ ap-ac } \\
$1.0 / 1.0$ & 6.6 & 15.6 & 19.4 & 5.8 & 2.74 \\
$1.8 / 1.8$ & 5.9 & 19.5 & 22.8 & 5.9 & 2.81 \\
$7.0 / 1.8$ & 6.2 & 19.0 & 22.4 & 5.9 & 2.91 \\
$37.5 / 1.8$ & 6.4 & 18.7 & 22.1 & 5.9 & 2.95 \\
\multicolumn{5}{c}{ abp-ac } \\
$1.0 / 1.0$ & 2.3 & 29.1 & 29.8 & 3.1 & 2.76 \\
$1.8 / 1.8$ & 1.4 & 38.2 & 38.5 & 2.6 & 2.57 \\
$7.0 / 1.8$ & 1.8 & 35.8 & 36.2 & 2.8 & 2.86 \\
$37.5 / 1.8$ & 2.2 & 33.7 & 34.2 & 3.0 & 3.00
\end{tabular}

${ }^{a}$ Based on continuum reaction field calculations at the AM1 CIS level $\mathrm{l}^{42-44}$ and GMH analysis. ${ }^{22-25}$ Corresponding vacuum ab initio and DFT results, and experimental results, are given in Tables 2 and 3.

charged) as well as significantly less interaction between the two ring systems in the ground state. Indeed, these assumptions are borne out in the calculations. A DFT optimization of the ground state leads to anthracene and phenyl rings at $90^{\circ}$ with respect to each other, indicating modest electronic coupling between the two subunits. In addition, the CT state is above $4.6 \mathrm{eV}$ at the ab initio CIS level and is at $4.3 \mathrm{eV}$ on the basis of the INDO/s (the corresponding state in ap-ac was at $2.3 \mathrm{eV}$ for the $\varphi=90^{\circ}$ geometry).

\section{Solvatochromic Trends and Non-Condon Behavior}

To provide insight into the influence of polar solvation on the optical CSh process (eq 4), we have carried out RF calculations at the CIS level using the AM1 Hamiltonian. ${ }^{42-44}$ Results for vertical absorption are presented (Table 7) and compared for the ab-ac and abp-ac systems (Figure 1), based on the ground-state geometries reported above. The new data extend the results of an earlier study limited to abp-ac and based on approximate molecular geometries. ${ }^{45}$

Trends with solvent polarity are assessed in terms of variation of transition energy $\Delta E_{12}$ (solvatochromism) and diabatic dipole shift $\left(\Delta \mu_{\mathrm{DA}}\right)$ and also the matrix elements $H_{\mathrm{DA}}$ and $\mu_{12}$ (nonCondon behavior). ${ }^{26,27}$ The calculated results help to provide a picture of the relative strength of solvation when the cationic charge is located at the D and A sites. The solvent polarity is represented here by an RF Hamiltonian based on a dielectric continuum model. ${ }^{45,46}$

4.1. Reaction Field Hamiltonian. In Franck-Condon controlled CT processes in solution, the total Hamiltonian may be expressed as a sum of three terms: the vacuum term and two solvent RF terms, corresponding, respectively, to fast electronic (denoted below as "optical") and slow nuclear (denoted below as "inertial") response of a solvent medium to the electronic process occurring in the solute (eq 8): ${ }^{45,46}$

$$
H=H_{\mathrm{vac}}+\left(H_{\mathrm{op}}\right)_{\mathrm{solv}}+\left(H_{\mathrm{in}}\right)_{\mathrm{solv}}
$$

For the vertical CSh process, the inertial RF term in eq 8 corresponds to the inertial response of the medium in equilibrium with the solute charge density prior to excitation. At the continuum level, this is governed by the inertial dielectric factor (the so-called Pekar factor), which may be expressed as the product of the inertial "dielectric constant", $\epsilon_{0}-\epsilon_{\infty}$, and the screening factor $\left(\epsilon_{0} \epsilon_{\infty}\right)^{-1}$, where $\epsilon_{0}$ and $\epsilon_{\infty}$ are, respectively, the static and optical dielectric constants.

Aside from the fast and slow time scale separation regarding the electronic and nuclear motion, in formulating the optical RF term, which involves only $\epsilon_{\infty}$ (and not $\epsilon_{0}$ ), we consider two different limiting cases of relative electronic time scales (i.e., the time scales of the solvent valence electrons and of the transferring electron (the "active" solute electron)). ${ }^{45,46}$ Since the CSh energy gaps in the present study are considerably smaller than the solvent CT energy gaps, we adopt the limit of fast solvent electronic response, an approach similar to the "BO" model of Kim and Hynes. ${ }^{47}$ This model has the advantage of yielding initial and final states that are orthogonal, since $\left(H_{\mathrm{el}}\right)_{\text {solv }}$ then depends only on the instantaneous solute density operator $\left(\rho_{\mathrm{op}}\right)$, in contrast to the more common mean field limit based on the charge density (the expectation value of $\rho_{\mathrm{op}}$ ).

The RF model described above has been implemented for use with the AM1 semiempirical Hamiltonian. ${ }^{48,49}$ The calculations employed a realistic solute cavity based on superposed atomic spheres, but differ from conventional polarized continuum models $(\mathrm{PCM})^{50}$ by incorporating distinct effective cavities for optical and inertial response (the so-called "frequency resolved cavity model"). ${ }^{51,52}$ In the discussion below we refer to a solvent with only optical response $\left(\epsilon_{0}=\epsilon_{\infty}\right)$ as "nonpolar", whereas "polar solvent" refers to the case of dipolar solvent molecules where $\epsilon_{0} \neq \epsilon_{\infty}$.

To highlight the role of solvation in CSh processes, eq 5 may be elaborated as follows:

$$
\Delta E_{12}=\left(\Delta G^{\circ}\right)_{\mathrm{vac}}+\lambda_{\mathrm{mol}}+\left(\Delta G^{\circ}\right)_{\mathrm{solv}}+\lambda_{\mathrm{solv}}
$$

where the first two terms are the vacuum contributions (including a molecular contribution to $\lambda$ assumed to be independent of the presence or absence of low-frequency inertial solvent polarization), the third term is the solvent contribution to the equilibrium free energy change (including both optical and inertial terms), and $\lambda_{\text {solv }}$ is the reorganization energy due to the inertial solvent polarization. For compactness, we suppress the "1,2" state subscripts for the $\Delta G^{\circ}$ and $\lambda$ quantities, and we refer below to $\left(\Delta G^{\circ}\right)_{\text {solv }}$ and $\left(\Delta G^{\circ}\right)_{\text {solv }}+\lambda_{\text {solv }}$ as, repectively, the adiabatic and vertical solvent contribution to the CSh free energy change. Since $\Delta E_{12}$ refers to a vertical process, it may be considered to be a free energy change (i.e., the entropy contribution is zero). ${ }^{53}$

4.2. Electronic States. To first approximation, the CSh process may be viewed as passage of an electron from the highest occupied molecular orbital (HOMO), localized on the arylamine donor (D) moiety (ap or abp), to the lowest unoccupied molecular orbital (LUMO), localized on the acridinium acceptor (A) moiety (ac), thereby neutralizing the initial state cationic charge at the ac site and creating the final-state ap or abp hole. The HOMO is primarily confined to the aminophenyl (ap) group in both ap-ac and abp-ac, but in abp-ac there is some delocalization onto the internal phenyl group. The LUMO is delocalized over the entire ac group. In the CIS calculations reported below, we included about 40 CI basis functions, composed of the SCF ground state (obtained in a vacuum) and single excitations from it. For a given solvent, these basis functions yield the adiabatic states (eigenfunctions of $H_{\mathrm{RF}}$ ) from 
which the two-state space for the GMH CSh analysis is defined as that spanned by the two lowest-energy eigenstates, selecting the two eigenfunctions dominated by the initial and final localized hole states. In all cases, this pair of states was found to yield a reasonable representation of the charge-localized diabatic states $\left(\mathrm{DA}^{+}\right.$and $\left.\mathrm{D}^{+} \mathrm{A}\right)$, while the next higher-lying eigenstate was associated with the locally excited acceptor state $\left(\mathrm{DA}^{+*}\right)$.

For a given diabatic basis, $\Delta \mu_{\mathrm{DA}}$ is by construction fixed, independent of variations in solvent polarization, whereas $H_{\mathrm{DA}}$ is expected in general to vary with solvent polarization (since the solvent-solute component of the Hamiltonian depends explicitly on this polarization), ${ }^{45,46}$ a manifestation of nonCondon behavior that we designate as a first-order effect. ${ }^{54}$ The consequences of solute polarizability must also be considered. This polarizability will cause the solute wave function and charge density to be modified in response to variation of the solvent polarization. A limited degree of polarizability is entailed in a two-state quantum mechanical model (e.g., as represented by eq 6). A more reliable treatment, however, is provided by a larger scale CI. As a result of this polarizability, the two-state space projected out of the larger space of CI eigenfunctions is not invariant, but rather is an effective "in situ" space that varies with the degree of solvent polarization. Thus, not only the adiabatic $\left(\Delta E_{12}, \Delta \mu_{12}\right.$, and $\left.\mu_{12}\right)$, but also the diabatic quantities $\left(H_{\mathrm{DA}}\right.$ and $\left.\Delta \mu_{\mathrm{DA}}\right)$ are expected to be solvent-dependent. In the case of $H_{\mathrm{DA}}$, this solute polarizability-induced non-Condon effect supplements the first-order effect noted above (in which the coupling varies with solvation for a fixed pair of diabatic states). ${ }^{54}$ In a recent theoretical study of the solvent dependence of the coumarin-153 line shapes employing a fixed two-state diabatic basis obtained from vacuum eigenstates, the solute polarizability was augmented by adding a classical auxiliary polarizability to a two-state quantum mechanical model. ${ }^{9}$

4.3. Calculations. In the calculations reported below for apac and abp-ac, the solvent is represented by four combinations of $\epsilon_{0}$ and $\epsilon_{\infty}$ : vacuum $\left(\epsilon_{0}=\epsilon_{\infty}=1\right)$; optical solvation $\left(\epsilon_{0}=\epsilon_{\infty}\right.$ $=1.8$, as in a totally nonpolar solvent); and polar solvation, with $\epsilon_{\infty}=1.8$ and $\epsilon_{0}=7$ (similar to the value for tetrahydrofuran) or 37.5 (representing acetonitrile). Since we are concerned here only with vertical absorption, we employ the optimized ground-state geometries discussed in Section 3.1. The torsional angles for the linkages of amine and aromatic moieties are given in Table 1.

4.4. Results. The various adiabatic and diabatic quantities for $\mathrm{ab}-\mathrm{ac}$ and abp-ac are listed in Table 7, displaying a number of systematic trends with increasing polarity. We note that, at the vacuum level, the AM1/CIS results differ somewhat from the INDO/s results (Tables 2 and 3), especially regarding the relative magnitudes of matrix elements $\left(H_{\mathrm{DA}}\right.$ and $\left.\mu_{12}\right)$. The main focus here is on variation of calculated properties with degree of solvation. In general, the ap-ac properties exhibit less variability than abp-ac, reflecting the stronger D/A coupling (due to closer proximity of D and A centroids) and, hence, less susceptibility to solvation in the former case.

For both CSh systems, $H_{\mathrm{DA}}$ and $\Delta \mu_{\mathrm{DA}}$ (as evaluated at the two-state GMH level, eq 6) are inversely related, with an increased separation of D/A centroids (measured by $\Delta \mu_{\mathrm{DA}}$ ) corresponding to a reduced electronic coupling $\left(H_{\mathrm{DA}}\right)$. The adiabatic dipole shift is always less than its diabatic counterpart, as expressed by eq $6 \mathrm{~b}$ and reflected in the data of Table 7 , which shows reduction of $20-25 \%$ (ap-ac) and $<5 \%$ (abp-ac), and the trend in $\Delta \mu_{12}$ magnitudes is seen to be the same as for $\Delta \mu_{\mathrm{DA}}$. For ab-ac, $\mu_{12}$ is roughly independent of solvent, whereas for
TABLE 8: Ap-ac: Calculated CSh Results and $\varphi$-Dependence, Using the KT-GMH Model ${ }^{a, b}$

\begin{tabular}{lcccc}
\hline geometry & $H_{\mathrm{DA}}\left(10^{3} \mathrm{~cm}^{-1}\right)$ & $\Delta \mu_{12}(\mathrm{D})$ & $\mu_{12}(\mathrm{D})$ & $\Delta E_{12}(\mathrm{eV})$ \\
\hline \multicolumn{5}{c}{ ab initio } \\
opt & 22.3 & 16.21 & 4.78 & 7.68 \\
$\Phi=90^{\circ}$ & 1.06 & 21.96 & 0.28 & 7.55 \\
ave (DFT) & 21.9 & 16.41 & 4.70 & 7.68 \\
ave (RHF) & 19.6 & 17.45 & 4.36 & 7.66 \\
\multicolumn{5}{c}{ INDO/s } \\
opt & 15.9 & 17.25 & 4.90 & 5.66 \\
$\Phi=90^{\circ}$ & 0.78 & 22.12 & 0.28 & 5.50 \\
ave (DFT) & 15.6 & 17.42 & 4.82 & 5.66 \\
ave (RHF) & 13.9 & 18.34 & 4.36 & 5.63 \\
experiment & 4.4 & 17.3 & 4.45 & 2.38
\end{tabular}

${ }^{a}$ Quantities as defined in Table 2, except that electronic states are expressed at the Koopmans' Theorem level (as described in Section 5). ${ }^{b} H_{\mathrm{DA}}$ is given by eq $12, \Delta \mu_{12}$ and $\mu_{12}$ are based on the HOMO/ LUMO orbitals, and $\Delta E_{12}$ is based on the HOMO/LUMO orbital energy difference.

abp-ac it follows the same trend as $H_{\mathrm{DA}}$. As anticipated above, the effective diabatic states are dependent on solvent (variable $\left.\Delta \mu_{\mathrm{DA}}\right)$, and $H_{\mathrm{DA}}$ exhibits an appreciable solvent-based nonCondon effect.

The quality of the two-state GMH model for the optical CSh process was assessed using the criterion developed by Rust et al. ${ }^{24}$ Their "smallness parameter" $\lambda_{\mathrm{D}}$ (a dimensionless quantity quite distinct from $\lambda$, as used in the present work to denote reorganization energy) was found to be similar to values obtained previously for the vacuum process (ranging from 0.1 for ap-ac to $0.2-0.3$ for abp-ac), thus supporting the utility of the two-state model in comparison to an extended three-state model including the locally excited (higher energy) acridinium state $\left(\mathrm{DA}^{+*}\right)$ discussed in Section 1. To provide additional information regarding the robustness of the two-state GMH model, we have evaluated the influence of the third state (i.e., $\left.\mathrm{DA}^{+*}\right)$ in terms of the "dressed" two-state model presented in ref 24 . At this level, the $H_{\mathrm{DA}}$ magnitudes are reduced by $\sim 20-$ $25 \%$ relative to the "bare" two-state results, while the $\Delta \mu_{\mathrm{DA}}$ magnitudes increased slightly $(\leq 3 \%)$.

4.4.1. Adiabatic Solvation. Before proceeding to discuss the full set of vertical CSh results, we comment first on the solvent contribution to the adiabatic CSh free energy change, $\left(\Delta G^{\circ}\right)_{\text {solv }}$ (as introduced in Section 4.1 and eq 9), based on separate equilibrium RF calculations for initial and final states. We find (results not shown explicitly) that $\Delta G^{\circ}$ decreases uniformly with polarity (i.e., $\epsilon_{0}$ ), while $\Delta \mu_{12}$ (or $\Delta \mu_{\mathrm{DA}}$, as discussed below) increases uniformly, the only exception being the very small increase in $\Delta G^{\circ}$ for ap-ac in going from vacuum to $\epsilon_{0}=1.8$ (Table 9). Both trends are dominated by solvation of the final hole state (i.e., at the ap or abp D site), implying a greater compactness of this site and/or its greater access to the solvent in comparison with the initial cationic charge (i.e., at the ac A site). Increased polarity causes the centroids of both the D and A orbitals to shift outward along the main axis (the vertical direction in Figure 1) within their respective moieties (i.e., ap or abp, and ac), but with the dominant shift at the D site. Note that for this comparison of separately equilibrated states, the GMH definition of diabatic states (and hence $\Delta \mu_{\mathrm{DA}}$ ) is not applicable when $\epsilon_{0} \neq \epsilon_{\infty}$, since GMH is based on a fixed set of coordinates (including solvent inertial modes) for both initial and final states. We thus rely on $\Delta \mu_{12}$ to provide estimates of the equilibrium $\mathrm{D}$ and $\mathrm{A}$ centroid shifts, when $\epsilon_{0} \neq \epsilon_{\infty}$.

The solvent contribution to the vertical and adiabatic CSh free energy shifts are the same for the case of nonpolar solvent (i.e., Table 7, when $\epsilon_{0}=\epsilon_{\infty}=1.8$ ), since $\lambda_{\text {solv }}=0$. The change 
TABLE 9: Hole Transfer in Ap-ac ${ }^{+}$: Calculated CSh Results and $\varphi$-Dependence, Using GMH at the KT and CIS Levels $^{a}$

\begin{tabular}{|c|c|c|c|c|}
\hline geometry & $H_{\mathrm{DA}}\left(10^{3} \mathrm{~cm}^{-1}\right)$ & $\Delta \mu_{12}(\mathrm{D})$ & $\mu_{12}(\mathrm{D})$ & $\Delta E_{12}(\mathrm{eV})$ \\
\hline \multicolumn{5}{|c|}{ KT } \\
\hline $10^{\circ}$ & 3.0 & 8.02 & 9.85 & 6.57 \\
\hline opt & 2.4 & 18.65 & 7.33 & 7.61 \\
\hline $90^{\circ}$ & 0.22 & 24.69 & 0.64 & 8.65 \\
\hline \multicolumn{5}{|c|}{ CIS } \\
\hline $10^{\circ}$ & 3.5 & 5.96 & 6.97 & 7.70 \\
\hline opt & 2.7 & 13.03 & 5.18 & 8.76 \\
\hline $90^{\circ}$ & 0.23 & 20.47 & 0.41 & 11.30 \\
\hline
\end{tabular}

${ }^{a}$ Comparison of INDO GMH results based on KT and CIS calculations for the CSh process in the ap-ac ${ }^{+}$dication. The KT-GMH one-electron quantities are based on the HOMO and HOMO-1 of the closed shell ap-ac SCF wave function.

from a small increase in $\Delta G^{\circ}$ for ap-ac to appreciable decrease for abp-ac (relative to vacuum results) suggests that the final state (D) hole in apb-ac is more accessible to solvent than it is in ap-ac, where it is in close proximity to the relatively bulky ac group. The observation that solvent polarity tends to reduce $\Delta G^{\circ}$ is compatible with conclusions reached in related experimental studies of CSh involving acridinium systems. ${ }^{21}$

The appreciable increase in $\Delta \mu_{\text {DA }}$ for ap-ac and apb-ac upon nonpolar solvation (Table $9, \epsilon_{0}=\epsilon_{\infty}=1.8$ ) provides an example of the influence of solute polarizability (of the type discussed in Section 4.2). This effect is due almost entirely (>95\%) to a centroid shift of the final-state hole (ap site) in going from vacuum to $\epsilon_{0}=1.8$.

4.4.2. Vertical CSh Including Inertial Solvation. Trends for variable inertial solvation (i.e., variable $\epsilon_{0}$, with $\epsilon_{\infty}$ fixed at 1.8) are seen to be monotonic for all quantities displayed in Table 7. In contrast to the adiabatic solvation results discussed in Section 4.4.1, we find an appreciable blue shift with increasing polarity, accompanied by a reduction in $\Delta \mu_{\mathrm{DA}}$ (and $\Delta \mu_{12}$ ). Increasing polarity causes the energy of both initial and final cationic states to drop, but the dominant effect is for the initial state, with which the inertial solvent polarization is in equilibrium. In terms of eq 9 , this means that the reduction of $\Delta G^{\circ}$ due to adiabatic solvation (as a result of preferential equilibrium solvation of the final hole state (D site), as discussed above) is more than offset by the increase in the nonequilibrium inertial term $\lambda_{\text {solv }}$, a positive definite quantity. The calculated blue shift with increasing solvent polarity is consistent with small blue shifts found experimentally. ${ }^{19}$

As in the adiabatic solvation discussed in Section 4.4.1, the final cationic state remains the more polarizable one, but in contrast to the adiabatic CSh case (where the final as well as the intial states involve equilibrium solvation), for vertical CSh the centroid of the final state hole shifts inward with increasing solvent polarity (i.e., away from the terminal $\mathrm{NH}_{2}$ group), leading to the observed overall reduction in $\Delta \mu_{\mathrm{DA}}$ (and $\Delta \mu_{12}$ ). Thus, whereas increased polarity of solvent in equilibrium with the final hole tends to "localize" this hole by pulling it outward, the opposite effect occurs when the solute polarizability responds to increasing polarity of a solvent in equilibrium with the other state in the CSh process (i.e., the initial one).

Corresponding to the reduction (with increasing solvent polarity) in separation of $\mathrm{D}$ and A centroids, $H_{\mathrm{DA}}$ (and also $\mu_{12}$ ) increases in magnitude, displaying a solvent-induced nonCondon behavior. The opposite trend has been found in calculations for vertical emission, in which the inertial polarization is in equilibrium with the hole state at the D site. ${ }^{45}$

5. KT-GMH Treatment of ap-ac. In the strict "one-electron" implementation of the KT-GMH model introduced in Section
2 , the many-electron quantity $H_{\mathrm{DA}}$ in eq $6 \mathrm{a}$ is replaced by its orbital counterpart, denoted here as $h_{\mathrm{DA}}$, in which $\Delta E_{12}$ is approximated by an orbital energy difference and all dipole quantites are replaced by orbital analogues (expectation values or transition moments). For the case of optical CSh (eq 4), the focus of the present article, the ground-state singlet can be represented as a closed-shell (single-determinant) ground state of the form (ignoring core orbitals):

$$
\Psi_{\mathrm{gs}}=A\left|\varphi_{1} \alpha \varphi_{1} \beta\right|
$$

while permutation symmetry leads to a two-determinant CSh final-state open-shell singlet of the form

$$
\psi_{\mathrm{CSh}}=A\left|\left(\varphi_{1} \varphi_{2}+\varphi_{2} \varphi_{1}\right) \alpha \beta\right| / \sqrt{ } 2
$$

As a result, we scale the strictly one-electron quantity $h_{\mathrm{DA}}$ by $\sqrt{ } 2$, yielding the approximate result

$$
H_{\mathrm{DA}}=\sqrt{ } 2 h_{\mathrm{DA}}
$$

As an alternative, one might consider scaling the transition dipole $\mu_{12}$ by $\sqrt{ } 2$, replacing $\mu_{12}$ by its scaled counterpart in the orbital analogue of eq 6 instead of scaling the overall $h_{\mathrm{DA}}$, as in eq 12. This approach yields results similar to those based on eq 12 , but is not considered further in the present work.

For the case of hole or electron transfer in $(n \pm 1)$-electron radical ion systems, in which the KT-GMH model is implemented using orbitals from the closed-shell $n$-electron SCF ground-state wave function, both initial and final states are represented by single determinants so that $h_{\mathrm{DA}}$ may be used directly.

Results for ap-ac are given in Table 8, based on the geometries used in Table 2 for the full GMH treatment. In modeling the CSh transition, we use the SCF HOMO and LUMO orbitals which are the dominant contributors to the description of the $\mathrm{CSH}$ in our CI results above. We present results at both ab initio and INDO levels. These results indicate that the trends observed in Table 2 are reproduced nicely using the KT-GMH model, but that there is an overestimation of the values of the coupling elements (by at most a factor of 2) and the adiabatic dipole moment differences. The overestimation of the coupling element is almost entirely connected with use of the LUMO and HOMO orbital energy differences to approximate the excitation energy. It is well-known that the virtual orbital energies are too high in the HF treatment, since virtual orbitals are defined in the field of $n$ rather than $n-1$ electrons, as would be the case for occupied orbitals. The orbital energy difference yields estimates of excitation energies $\left(\Delta E_{12}\right)$ high by about a factor of 2 , and this translates into a high value for the coupling element. The adiabatic dipole differences are large as well (when compared with the CI values) since the field defining the LUMO at the SCF level has a doubly occupied HOMO, and will allow less delocalization than when the excitation is treated properly at the CI level. Nevertheless, the trends obtained from the method are excellent, and to within a factor of 2 the coupling elements based on eqs $10-12$ are quite satisfactory.

An improved orbital estimate for $\Delta E_{12}$ may be obtained from triplet state calculations in which both orbitals have the same occupation in the SCF wave function. Test calculations were carried out based on the lowest triplet state of ap-ac, using the INDO restricted open shell SCF (ROHF) triplet orbitals and their associated energies and dipole moments to calculate the coupling. In comparison with the results based on the singlet 
closed-shell reference wave function (Table 8), these calculations yielded a value of the electronic coupling at the equilibrium geometry $\left(6640 \mathrm{~cm}^{-1}\right)$ significantly closer to the all-electron value from Table 2 at the INDO S/CI level.

To further test the KT-GMH method, we applied it to hole transfer in ap-ac (Table 9). In this case, we treat ap-ac ${ }^{+}$(which possesses a double positive charge, since ap-ac is itself positively charged) and, at the one-electron level, characterize the transfer as occurring between the HOMO and HOMO-1 orbitals of the ap-ac closed-shell system. These orbitals, which are localized, respectively, on the phenyl and acridinium subunits, were obtained from ground-state INDO/s SCF ap-ac calculations. In the full GMH calculations for ap-ac ${ }^{+}$, we employed a CI exactly analogous to that used for ap-ac, based on the ground-state SCF ap-ac orbitals. It is seen that the agreement between the GMH and KT-GMH coupling elements is remarkably good, generally within $10 \%$ of the full GMH result, supporting the notion that correlation has little effect on off-diagonal matrix elements of the Hamiltonian. Voityuk and Rösch found excellent agreement between the KT-GMH method and their FCD approach for hole transfer in DNA base pairs. ${ }^{33}$ The adiabatic dipole moment differences are still somewhat large. Overall, it would appear that the KT-GMH is a quick means for getting reasonable estimates of the electronic coupling element based on a single SCF calculation. Note that just as for the full GMH treatment, there is no need to apply an external field or adjust geometries to achieve near degeneracy, and that a variety of CT-type interactions can be treated at once.

\section{Summary}

We have presented results of calculations for various properties of the ground and CSh excited states of several arylaminesubstituted acridinium ion systems that have been studied experimentally. Using ab initio and semiempirical quantum mechanical methods (CIS) together with the GMH model, we examined the excitation energies, dipole moment shifts, and electronic coupling elements for the vertical CSh processes in these systems. We have also examined solvent effects on these properties using a dielectric continuum reaction field model. In conformity with the previous picture for understanding the acridinium systems, we find the three lowest-energy eigenstates (in order of increasing energy) to be ground $\left(\mathrm{DA}^{+}\right), \mathrm{CSh}\left(\mathrm{D}^{+} \mathrm{A}\right)$, and locally excited $\left(\mathrm{DA}^{+*}\right)$ states.

Ground-state geometries have been optimized in vacuo for four different acridinium systems using DFT, and these geometries were used in the CSH studies (thermal averaging over a Boltzmann distribution of torsion angles had little effect on calculated vertical CSh properties). Of particular interest for electronic coupling are the torsional angles linking the acridinium and arylamine $(\varphi)$ and the aryl and amine $(\omega)$ moieties. Optimal ground state $\varphi$ values reflect a large degree of twisting away from planarity $\left(52-56^{\circ}\right)$, comparable to the large values obtained from crystal structure data. The CSh calculations indicate that there is strong electronic coupling in these systems over a wide range of torsional angles. Nevertheless, the initial and final cationic states remain reasonably well-localized over this range (as a result of the sizable energy gaps), being roughly confined, respectively, to the acridinium and arylamine portions of the molecules. Twisting of the ap-ac amino group toward the perpendicular conformation $\left(\omega=90^{\circ}\right)$ relative to the equilibrium value caused a rise in the in vacuo energy for both ground and CSh states and also a reduction of $\Delta \mu_{12}$, thus indicating little likelihood of a TICT effect in polar media. As an additional test (to explore the distinction between CSh and
CS processes), isoelectronic replacement of the acridinium $\mathrm{N}^{+}$ atom by a neutral $\mathrm{C}$ atom was found to cause a pronounced increase of the optical excitation energy, an increase in degree of charge localization, and an equilibrium $\varphi$-value near $90^{\circ}$. For the biphenylamine donor (abp-ac), an additional torsional degree of freedom arises $\left(\gamma=30^{\circ}\right.$ in the ground state).

The calculated in vacuo properties are in generally reasonable accord with the qualitative features of available spectral data. The best correspondence is obtained from INDO/s calculations: large coupling (several thousand $\mathrm{cm}^{-1}$, with INDO/s greater than experimental estimate by factors of 1.3 to 2); the same trend as experiment for $\Delta \mu_{12}$ (INDO/s less by up to $20 \%$ ); and $\Delta E_{12}$ values in close correspondence (within $0.25 \mathrm{eV}$ ). The ab initio CIS results, in comparison with those from experiment and INDO/s, gave appreciably larger coupling values, smaller dipole shifts $\left(\Delta \mu_{12}\right)$, and larger excitation energies. Consistent with results from other groups, we found that TDDFT gave excitations energies that were in serious disagreement with experiment for weakly interacting CT states.

Variation of electronic properties of the CSh process with solvent polarity for two of the acridinium species (ap-ac and abp-ac) has been monitored with AM1/CIS dielectric continuum reaction field calculations, based on a model corresponding to the limiting case of instantaneous response of solvent electrons to the transferring solute electron. Solvation is found to be dominated by the donor site: that is, the final hole state $\left(D^{+} A\right)$ is more effectively solvated than the initial cationic state $\left(\mathrm{DA}^{+}\right)$. Thus, polar solvation reduces the adiabatic CSh energy gap $\left(\Delta G^{\circ}\right)$ relative to the vacuum value. For vertical $\mathrm{CSh}$, however, the CSh excitation energy is blue-shifted with increasing polarity, a consequence of the fact that the solvent polarization, equilibrated to the initial state, is increasingly less favorable for final state solvation. The diabatic quantities $H_{\mathrm{DA}}$ and $\Delta \mu_{\mathrm{DA}}$ also vary with solvent polarity, reflecting a solvent-driven nonCondon effect arising from the influence of solute polarizability.

Finally, a version of the GMH model based on Koopmans' Theorem has been developed and found to yield coupling elements generally within a factor of 2 of the many-electron GMH for a sample acridinium system (ap-ac), but with overestimated adiabatic and diabatic dipole moment differences. Further tests of the method will be required to establish its general utility, but the current results suggest it may be a simple means of exploring trends in the coupling element in significantly larger systems than are currently amenable to the GMH method based on use of correlated electronic structure theory approaches.

Acknowledgment. We are indebted to Professor G. Jones (Boston University) for stimulating this study in the course of many valuable discussions and for providing us with unpublished spectral and kinetic data generated by him and his students. We are also indebted to Drs. Fred Vance and Robert Johnson (Northwestern University) for making available the unpublished Stark data cited in ref 37 and to Professor J. Hupp (Northwestern University) for several discussions concerning these data. R.J.C. gratefully acknowledges financial support from the National Science Foundation (CHE-9731634, CHE-0353199) and the donors of the Petroleum Research Fund. M.D.N. was supported by the Division of Chemical Sciences, U.S. Department of Energy, under Grant DE-AC02-98CH10886.

Supporting Information Available: Equilibrium geometries of all acridinium systems, obtained at the DFT level (B3LYP) using the $6-31 \mathrm{G}^{*}$ basis. This material is available free of charge via the Internet at http://pubs.acs.org. 


\section{References and Notes}

(1) Electron Transfer in Chemistry; Balzani, V., Ed.; Wiley-VCH: Weinheim, Germany, 2001; Vols. I-V.

(2) Davis, W. B.; Ratner, M.; Wasielewski, M. R. J. Am. Chem. Soc. 2001, 123, 7877.

(3) Newton, M. D. Coord. Chem. Rev. 2003, 238, 167.

(4) Miller, J. R.; Calcaterra, L. T.; Closs, G. L. J. Am. Chem. Soc. 1984, 106, 3047.

(5) Jones, G.; Farahat, M. S.; Greenfield, S. R.; Gosztola, D. J.; Wasielewski, M. R. Chem. Phys. Lett. 1994, 229, 40.

(6) Reichardt, C. Chem. Rev. 1994, 94, 2319.

(7) Reichardt, C. Solvents and Solvent Effects in Organic Chemistry, 2nd ed.; VCH: Weinheim, Germany, 1968.

(8) Grabowski, Z. R.; Rotkiewicz, K.; Rettig, W. Chem. Rev. 2003, 103,3899 . 8516.

9) Matyushov, D. V.; Newton, M. D. J. Phys. Chem. A 2001, 105,

(10) Gould, I. R.; Noukakis, D.; Gomez-Jahn, L.; Goodman, J. L.; Farid, R. S. J. Am. Chem. Soc. 1993, 115, 4405.

(11) A two-sphere Born model for CSh processes would be analogous to (but distinct from) the Weller equation (Weller, A. Z. Phys. Chem. 1982 $133,93)$, used for CS processes in which the point dipole model is not suitable. However, in the CSh case, the two-center Coulombic term is absent and both initial and final states are solvated.

(12) Gould, I. R.; Noukakis, D.; Gomez-Jahn, L.; Young, R. H.; Goodman, J. L.; Farid, S. Chem. Phys. 1993, 176, 439.

(13) Marcus, R. A.; Sutin, N. Biochim. Biophys. Acta 1985, 811, 265.

(14) Marcus, R. A. J. Chem. Phys. 1956, 24, 966.

(15) Kakitani, T.; Mataga, N. J. Phys. Chem. 1986, 90, 993.

(16) Kakitani, T.; Mataga, N. J. Phys. Chem. 1987, 91, 6277.

(17) Marcus, R. A. J. Phys. Chem. 1989, 93, 3078.

(18) Gould, I. R.; Moser, J. E.; Armitage, B ; Farid, R. S.; Goodman, J. L.; Herman, M. S. J. Am. Chem. Soc. 1989, 111, 1917.

(19) Jonker, S. A.; Ariese, F.; Verhoeven, J. W. Recl. Trav. Chim. PaysBas 1989, 108, 109 .

(20) Jones, G.; Yan, D. X.; Greenfield, S. R.; Gosztola, D. J.; Wasielewski, M. R. J. Phys. Chem. A 1997, 101, 4939.

(21) Related data for intermolecular CSh in acridinium-based systems have been reported by Fukuzumi, S.; Ohkubo, K.; Suenobu, T.; Kato, K.; Fujitsuka, M.; Ito, O. J. Am. Chem. Soc. 2001, 123, 8459 .

(22) Cave, R. J.; Newton, M. D. Chem. Phys. Lett. 1996, 249, 15.

(23) Cave, R. J.; Newton, M. D. J. Chem. Phys. 1997, 106, 9213.

(24) Rust, M.; Lappe, J.; Cave, R. J. J. Phys. Chem. A 2002, 106, 3930.

(25) Newton, M. D. In Electron Transfer in Chemistry; Balzani, V., Ed.; Wiley-VCH: Weinheim, Germany, 2001; Vol. I, p 3.

(26) Onuchic, J. N.; Beratan, D. N.; Hopfield, J. J. J. Phys. Chem. 1986, $90,3707$.

(27) Medvedev, E. S.; Stuchebrukhov, A. A. J. Chem. Phys. 1997, 107, 3821.

(28) Newton, M. D. Chem. Rev. 1991, 91, 767.

(29) Frisch, M. J.; Trucks, G. W.; Schlegel, H. B.; Scuseria, G. E.; Robb,

M. A.; Cheeseman, J. R.; Zakrzewski, V. G.; Montgomery, J. A., Jr.;
Stratmann, R. E.; Burant, J. C.; Dapprich, S.; Millam, J. M.; Daniels, A. D.; Kudin, K. N.; Strain, M. C.; Farkas, O.; Tomasi, J.; Barone, V.; Cossi, M.; Cammi, R.; Mennucci, B.; Pomelli, C.; Adamo, C.; Clifford, S.; Ochterski, J.; Petersson, G. A.; Ayala, P. Y.; Cui, Q.; Morokuma, K.; Malick, D. K.; Rabuck, A. D.; Raghavachari, K.; Foresman, J. B.; Cioslowski, J.; Ortiz, J. V.; Stefanov, B. B.; Liu, G.; Liashenko, A.; Piskorz, P.; Komaromi, I.; Gomperts, R.; Martin, R. L.; Fox, D. J.; Keith, T.; Al-Laham, M. A.; Peng, C. Y.; Nanayakkara, A.; Gonzalez, C.; Challacombe, M.; Gill, P. M. W.; Johnson, B. G.; Chen, W.; Wong, M. W.; Andres, J. L.; Head-Gordon, M.; Replogle, E. S.; Pople, J. A. Gaussian 98, revision A.11.3; Gaussian, Inc.: Pittsburgh, PA, 1998

(30) Zerner, M. C.; Loew, G. H.; Kirchner, R. F.; Mueller-Westerhoff, U. T. J. Am. Chem. Soc. 1980, 102, 589.

(31) Zerner, M. C. ZINDO: A Comprehensive Semiempirical SCF/CI Package, version 3.7; Molecular Simulations Inc.: San Diego, CA, 1991

(32) Koopmans, T. Physica 1933, 1, 104

(33) Voityuk, A. A.; Rosch, N. J. Chem. Phys. 2002, 117, 5607.

(34) Goubitz, K.; Reiss, C. A.; Heijdenrijk, D.; Jonker, S. A.; Verhoeven, J. W. Acta Crystallogr., Sect. C 1989, 45, 1348.

(35) Bastiansen, O.; Samdal, S. J. Mol. Struct. 1985, 128, 115.

(36) Rapid reverse CSh (as well as forward photoinitiated CSh) kinetics in a polar solvent has been reported in ref 5 .

(37) Vance, F.; Johnson, R.; Hupp, J. T. Unpublished measurement of optical absorption in methanol solution and Stark spectra in 2-methyl tetrahydrofuran/butyronitrile glasses.

(38) Casida, M. E. In Recent Developments and Applications in Density Functional Theory; Seminario, J. M., Ed.; Elsevier: Amsterdam, 1996.

(39) Cai, Z.-L.; Sendt, K.; Reimers, J. R. J. Chem. Phys. 2002, 117, 5543.

(40) Dreuw, A.; Head-Gordon, M. J. Am. Chem. Soc. 2004, 126, 4007.

(41) Tozer, D. J. J. Chem. Phys. 2003, 119, 12697.

(42) Dewar, M. J. S.; Zoebisch, E. G.; Healy, E. F.; Stewart, J. J. P. J. Am. Chem. Soc. 1985, 107, 3902.

(43) Soujanya, T.; Saroja, G.; Samanta, A. Chem. Phys. Lett. 1995, 236, 503 .

(44) Gorse, A.-D.; Pesquer, M. J. Phys. Chem. 1995, 99, 4039.

(45) Newton, M. D. Theor. Chem. Acc. 2003, 110, 307.

(46) Basilevsky, M. V.; Chudinov, G. E.; Newton, M. D. Chem. Phys

1994, 179, 263.

(47) Kim, H. J.; Hynes, J. T. J. Chem. Phys. 1992, 96, 5088.

(48) Newton, M. D. In Molecules as Components of Electronic Devices; Lieberman, M., Ed.; ACS Symposium Series 844; American Chemical Society: Washington, DC, 2003; p 196.

(49) Leontyev, I. V.; Basilevsky, M. V.; Newton, M. D. Theor. Chem. Acc. 2004, 111, 110.

(50) Tomasi, J.; Persico, M. Chem. Rev. 1994, 94, 2027.

(51) Basilevsky, M. V.; Rostov, I. V.; Newton, M. D. Chem. Phys. 1998, $232,189$.

(52) Newton, M. D.; Basilevsky, M. V.; Rostov, I. Chem. Phys. 1998 232, 210.

(53) Marcus, R. A.; Sutin, N. Comments Inorg. Chem. 1986, 5, 119.

(54) Kuznetsov, A. M. Nouv. J. Chim. 1981, 5, 427. 